\title{
Design and fabrication of binary slanted surface-relief gratings for a planar optical interconnection
}

\author{
J. Michael Miller, Nicole de Beaucoudrey, Pierre Chavel, Jari Turunen, \\ and Edmond Cambril
}

\begin{abstract}
We propose the use of binary slanted surface-relief gratings with parallel-groove walls as input and output couplers in a planar optical interconnect. Parametric optimization of cascaded output couplers is employed to design an interconnect consisting of $N$ output couplers producing a uniform intensity distribution with a high efficiency that may be realized in one lithographic etching step. The sensitivity of a $N=4$ interconnect to various fabrication errors is analyzed. We demonstrate the operation of a slanted surface-relief grating manufactured with electron-beam lithography and reactive-ion etching for an operating wavelength of $\lambda=0.633 \mu \mathrm{m}$. (C) 1997 Optical Society of America

Key words: Subwavelength gratings, grating couplers, slanted surface-relief profiles, planar optics.
\end{abstract}

\section{Introduction}

Planar-integrated optical systems ${ }^{1}$ are of considerable interest in optical interconnection since the optical components in these miniature systems are inherently aligned. ${ }^{2}$ The constituent optical elements in planar optical systems are typically surfacerelief diffractive components. ${ }^{1}$ Microlithographic fabrication techniques are employed to generate these structures, which may have a binary, multilevel, or continuous profile. The synthesis of these diffractive elements is often based on the paraxial approximation, which leads to profiles with transverse features of dimensions much larger than the wavelength of the incident light $\lambda$. If nonparaxial deflection angles are required, as is the case in planar optics, the diffractive profile will contain features with dimensions of the order of the optical wavelength $\lambda$. Consequently, the diffraction characteris-

J. M. Miller, N. de Beaucoudrey, and P. Chavel are with the Institut d'Optique Théorique et Appliquée, Centre National de la Recherche Scientifique, B.P. 147, 91403 Orsay, France. J. Turunen is with the Department of Physics, University of Joensuu, P.O. Box 111, FIN-80101 Joensuu, Finland. E. Cambrill is with the Laboratoire de Microstructures et de Microélectronique, Centre National de la Recherche Scientifique, 196 avenue Henri Ravera, B.P. 107, 92225 Bagneaux, France.

Received 12 November 1996; revised manuscript received 27 March 1997.

0003-6935/97/235717-11 $\$ 10.00 / 0$

(C) 1997 Optical Society of America tics must be analyzed by use of electromagnetic theory. ${ }^{3}$ Furthermore, the use of resonance-domain diffractive elements ${ }^{4}$ in planar optics may alleviate some of the reported disadvantages of paraxial designs. ${ }^{5}$ This has only recently become possible with the rapid advances in submicrometer lithography and mathematical synthesis techniques in diffractive optics. ${ }^{6}$

In this paper we concentrate on the design of binary slanted surface-relief diffractive elements with subwavelength features for use in a planar optical backplane. The operation is based on input and output grating couplers, and the interconnection is established by multiple total internal reflections inside the backplane substrate, as illustrated in Fig. 1. In this ideal configuration the input coupler is designed to deflect normally incident light efficiently into the backplane substrate at angles larger than the critical angle, whereas the cascaded output couplers ensure a uniform intensity distribution with a high efficiency among the different processors. The main design problem of obtaining a large deflection for a normally incident wave can be mitigated by use of highfrequency binary lamellar gratings only with great difficulty. ${ }^{7,8}$ Likewise, multilevel gratings ${ }^{9}$ prove equally troublesome, since the blaze effect of triangular profiles is not valid in the resonance domain. Slanted volume holographic Bragg gratings ${ }^{10,11}$ would provide the ideal solution, but ultimately replication techniques will be used to mass-produce a 
(a)

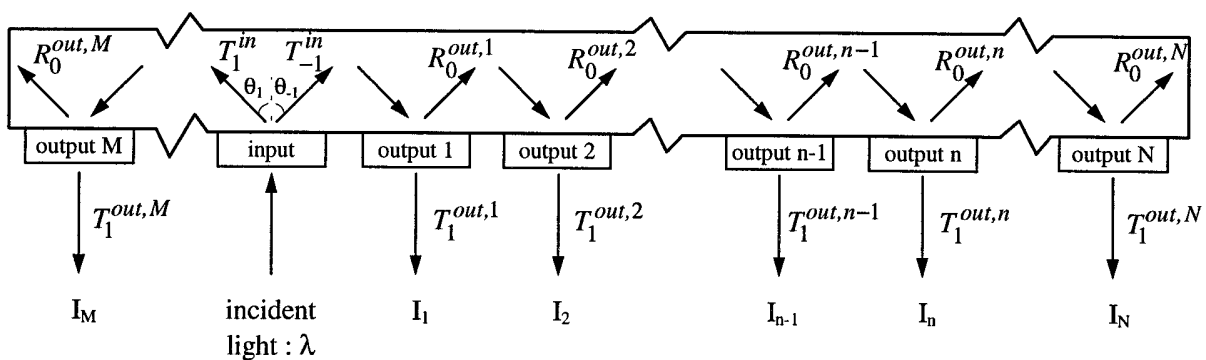

(b)

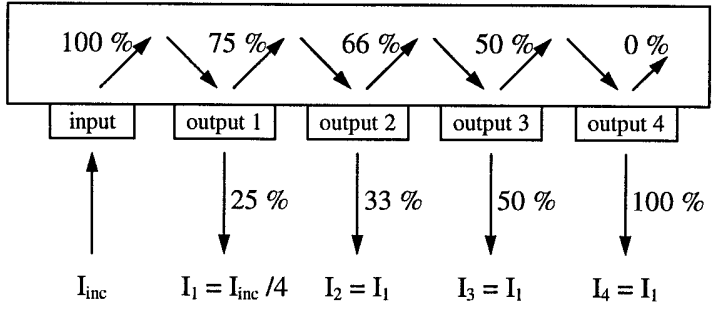

Fig. 1. (a) General configuration for a planar optical backplane interconnection. (b) Ideal $T_{1}^{\text {out, }, n}$ and $R_{0}^{\text {out }, n}$ for a system with $N=4$ and $M=0$.

planar optical back-plane, which clearly favors the use of some form of surface-relief diffractive element.

With the exception of a proposed backplane bus based on volume holographic gratings ${ }^{12}$ and holographically recorded surface-relief gratings, ${ }^{13}$ the demonstrator systems manufactured to date have employed identical binary lamellar surface-relief output-coupling gratings. ${ }^{14,15}$ If such a system is employed to distribute a clock signal in a multiprocessor system, one drawback is that the intensity at each processor diminishes monotonically with increased distance from the input coupler. This nonuniformity dephases the individual processor clocks, i.e., it introduces a clock skew. We can avoid this clock skew by designing individually the successive output-coupling gratings so that, in a cascaded formation, they produce a uniform intensity distribution.

In considering the multiple output couplers of Fig. 1 , the groove-slant angle of a binary grating can be employed as a new degree of freedom in the design process. The remaining grating parameters are then equal for each output coupler. A continuous choice of the diffraction-order efficiency is provided at each output coupler by variation of the slant angle. Some preliminary results with a variation of the slant angle were presented in Refs. 16 and 17 . Although such a combination of gratings provides an elegant solution to the problem of obtaining a uniform intensity distribution, the disadvantage is that one lithographic etching step is required to generate the surface-relief profile of each single output-coupler grating.

In what follows, we further elaborate the design of these slanted surface-relief gratings such that only a single etching step is needed to produce $N$ output couplers, which yield a uniform intensity distribution and a high efficiency. By utilizing parametric optimization, we design a series of $N=4$ and $N=8$ such couplers with different fill factors. The sensitivity of the $N=4$ solution is analyzed in the presence of the most prevalent fabrication errors. Finally, we demonstrate the operation of a slanted surface-relief grating coupler at $\lambda=0.633 \mu \mathrm{m}$. This component was realized in photoresist by use of direct electron-beam writing and reactive-ion etching (RIE). To our knowledge this is the first time that a slanted surface-relief grating has been used in a planar optic configuration.

\section{Design of the Grating Couplers}

We begin by determining the goal performance characteristics for input and output couplers in a perfect backplane interconnection. This is followed by a description of binary slanted surface-relief gratings and the optimization technique that we employ to implement the matching of the ideal grating-coupler attributes with such profiles.

\section{A. Optical Backplane}

The general configuration for a planar optical interconnection is illustrated in Fig. 1(a). Here the incident light is coupled into the backplane by means of the $l= \pm 1$ transmitted diffraction orders of the input grating coupler, making an angle $\theta_{ \pm 1}$ to the substrate normal. On either side of this grating reside $M$ and $N$ output couplers, respectively. We require the input coupler to have a splitting ratio that ensures an equal distribution of the incident light between the $M+N$ output couplers. Concentrating now on one side of the input coupler, the $k$ th reflected and the $l$ th transmitted diffraction-order efficiencies for the $n$th 
(a)

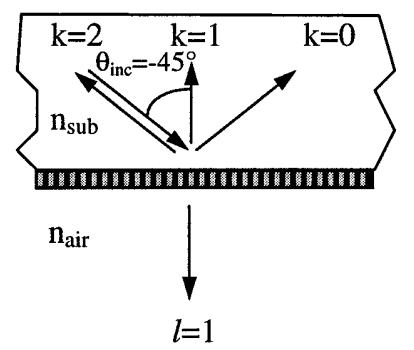

(b)

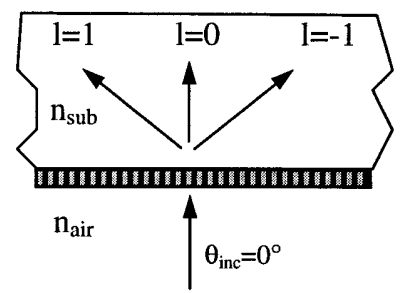

$\lambda$

Fig. 2. Grating geometry for an optical backplane: propagating orders for (a) an output and (b) an input coupler.

output coupler are denoted by $R_{k}^{\text {out, }, n}$ and $T_{l}^{\text {out, }, n}$. Multiple or single connections at the $n$th output may be envisaged. The useful diffraction orders then correspond either to several or to a lone propagating transmitted order. Here, only one connection at each output is considered. This corresponds to tailoring the diffraction-order efficiencies $T_{1}^{\text {out, } n}$ and $R_{0}^{\text {out, } n}$. To achieve a uniform distribution of the intensities emanating from each output coupler (i.e., $I_{n}$ $=I_{1}$ for $n=2, \ldots, N$ ), combined with a high efficiency, the diffraction-order efficiencies for the $N$ output couplers must follow the relations ${ }^{18}$

$$
T_{1}^{\text {out }, n}=(N-n+1)^{-1}, \quad R_{0}^{\text {out }, n}=1-T_{1}^{\text {out }, n},
$$

where $T_{l \neq 1}^{\text {out }, n}=R_{k \neq 0}^{\text {out, } n}=0$ and $n=1, \ldots, N$. The diffraction-order efficiencies $T_{1}^{\text {out }, n}$ and $R_{0}^{\text {out }, n}$ are shown in Fig. 1(b) for an ideal system with $N=4$ output couplers.

Turning our attention to the backplane characteristics, we see that the bounce angle $\theta_{B}$ inside the substrate must be larger than the critical angle $\theta_{\text {crit }}=$ $\arcsin \left(n_{\text {air }} / n_{\text {sub }}\right)$, where $n_{\text {sub }}$ represents the refractive index of the substrate. We set $\theta_{B}=45^{\circ}$, which is appropriate as soon as $n_{\text {sub }}>1.41$. For the component output couplers of the backplane [Fig. 2(a)], we fix the angle of the first transmitted diffraction order to $\theta_{1}^{\text {out }}=0^{\circ}$. The continental European form of the grating equation ${ }^{19}$ implies the following relation between the angle of incidence $\theta_{\text {inc }}$, the wavelength $\lambda$, the refractive index of the substrate $n_{\text {sub }}$, and the spatial period of the grating $\Lambda_{x}$ :

$$
\Lambda_{x}=-\frac{\lambda}{n_{\mathrm{sub}} \sin \theta_{\mathrm{inc}}} .
$$

Setting $\theta_{\text {inc }}=-\theta_{B}=-45^{\circ}$ and $n_{\text {sub }}=1.5$, we obtain $\Lambda_{x} / \lambda=0.943$. This result implies that the $k=1$ reflected order is also perpendicular to the substrate, whereas the $k=2$ reflected order becomes collinear to the incident direction. Similarly, for an input coupler with $\Lambda_{x} / \lambda=0.943$ [see Fig. 2(b)] and that is illuminated at normal incidence, the $l= \pm 1$ transmitted orders have diffraction angles of $\pm \theta_{B}$.

The design problem for the output couplers consists of searching for the optimal values of the useful diffraction-order efficiencies [given by Eqs. (1)] while minimizing the diffraction efficiencies of the unwanted propagating orders, i.e., the $k=1$ and $k=2$ reflected orders. Since the light intensity reaching each grating depends on the response of the previous gratings, we must consider a global synthesis problem with respect to the characteristics of the backplane, i.e., the $N$ cascaded output couplers. Accordingly, we employ the following figures of merit to quantify the backplane performance:

\section{The total efficiency}

$$
\eta=\sum_{n=1}^{N}\left(R_{0}^{\text {out }, n-1} \times R_{0}^{\text {out }, n-2} \times \cdots \times R_{0}^{\text {out }, 0}\right) T_{1}^{\text {out }, n},
$$

where we define $R_{0}^{\text {out, } 0}$ as the input efficiency onto the first output coupler. In the synthesis problem we require at least $\eta>90 \%$ when $R_{0}^{\text {out }, 0}=100 \%$.

2 . The uniformity error $U$, defined in terms of the intensities as

$$
U=\frac{I_{n}^{\max }-I_{n}^{\min }}{I_{n}^{\max }+I_{n}^{\min }}
$$

where $I_{n}^{\max }$ and $I_{n}^{\min }$ denote the maximum and minimum intensities, respectively, among the $N$ output beams. Typically, this value should be no higher than, say, $U=10 \%$, to avoid clock skew.

3. The overall efficiencies lost into the $k=1$ and $k=2$ unwanted reflected orders, given by

$$
\begin{aligned}
& \eta_{1, \text { lost }}=\sum_{n=1}^{N}\left(R_{0}^{\text {out }, n-1} \times R_{0}^{\text {out }, n-2} \times \cdots \times R_{0}^{\text {out }, 0}\right) R_{1}^{\text {out }, n}, \\
& \eta_{2, \text { lost }}=\sum_{n=1}^{N}\left(R_{0}^{\text {out }, n-1} \times R_{0}^{\text {out }, n-2} \times \cdots \times R_{0}^{\text {out }, 0}\right) R_{2}^{\text {out }, n},
\end{aligned}
$$

which must be as low as possible in order to avoid cross talk in the system.

It should be mentioned that surface-relief gratings with a sinusoidal profile and varying relief depths can give a uniform intensity distribution. ${ }^{13}$ In spite of the relatively straightforward nature of generating sinusoidal surface-relief profiles, a backplane based on these coupling gratings will have a low efficiency since large amounts of light are diffracted into the $k=2$ reflected order of the output couplers.

\section{B. Slanted Binary Surface-Relief Gratings}

Our selection of a surface-relief profile for the output couplers depends first on whether $N$ cascaded ele- 


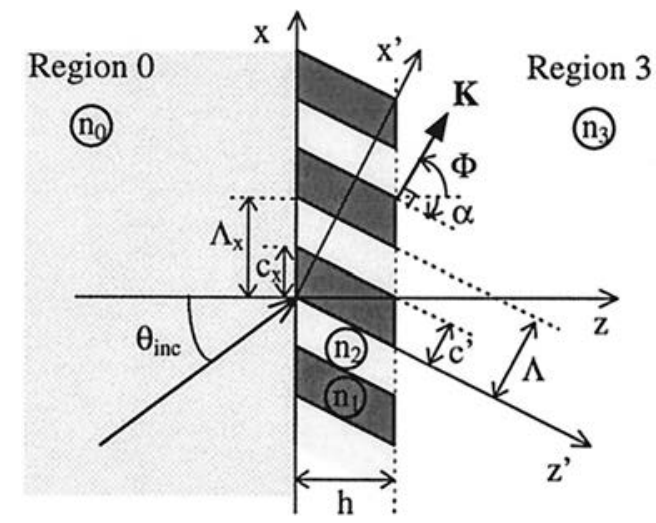

Fig. 3. Configuration for a slanted surface-relief grating.

ments can match the desired performance requirements (see Subsection 2.A) and second, whether we can realistically conceive of manufacturing such a multiple-grating system, i.e., the surface-relief profiles must be compatible with state-of-the-art microlithographic fabrication techniques. This latter requisite can be met if we employ gratings with single grooves of similar dimensions (thereby facilitating the control of proximity effects with electron-beam writing) and equal relief depths. The degrees of freedom in the design process then become the groove width and the relief depth, and, furthermore, we liberate the slant angle of the groove so that we are not confined to binary lamellar profiles.

In Fig. 3 is illustrated such a binary slanted surface-relief grating. ${ }^{16,17}$ A plane electromagnetic wave is incident upon the permittivity profile, which has the form

$$
n^{2}\left(x^{\prime}\right)= \begin{cases}n_{1}{ }^{2}, & \text { when } 0 \leq x^{\prime}<c^{\prime} \\ n_{2}{ }^{2}, & \text { when } c^{\prime} \leq x^{\prime}<\Lambda\end{cases}
$$

when $0 \leq z<h$. Here $\Lambda$ is the grating period in $x^{\prime}$ direction, $h$ is the relief depth, $\alpha$ denotes the slant angle, and $\mathbf{K}$ is the grating vector, which makes an angle $\Phi=\pi / 2+\alpha$ with the $z$ axis. The grating profile is characterized in the $(x, z)$ coordinate system by a single groove of width $c_{x}=c^{\prime} / \cos \alpha$, a period of $\Lambda_{x}=\Lambda / \cos \alpha$, and a fill factor of $f f=c_{x} / \Lambda_{x}$. Regions 0 and 3 consist of isotropic materials with permittivities $n_{0}^{2}$ and $n_{3}^{2}$.

Table 1. High-Efficiency Output-Coupler Grating Parameters and Diffraction Efficiencies in TE and TM Polarizations with a Constant Fill Factor $\mathrm{ff}_{\boldsymbol{n}}$

\begin{tabular}{ccc}
\hline $\begin{array}{c}\text { Parameters and } \\
\text { Diffracted Orders }\end{array}$ & TE Polarization & TM Polarization \\
\hline$h / \lambda$ & 1.058 & 2.351 \\
$\alpha$ & $-30.0^{\circ}$ & $-30.4^{\circ}$ \\
$f f$ & 0.5 & 0.5 \\
$T_{1}^{\text {out }, 4}(\%)$ & 97.2 & 97.6 \\
$R_{0}^{\text {out }, 4}(\%)$ & 0.1 & 0.6 \\
$R_{1}^{\text {out }, 4}(\%)$ & 0.4 & 0.9 \\
$R_{2}^{\text {out }, 4}(\%)$ & 2.3 & 0.8 \\
\hline
\end{tabular}

(a)

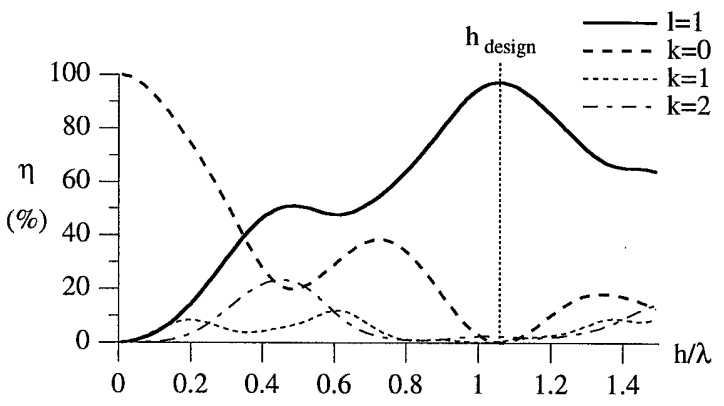

(b)

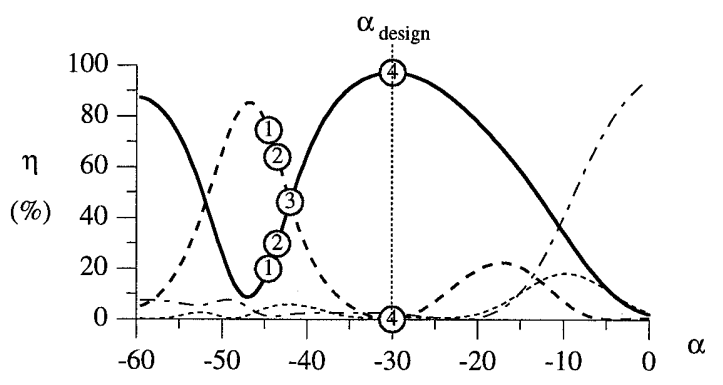

(c)

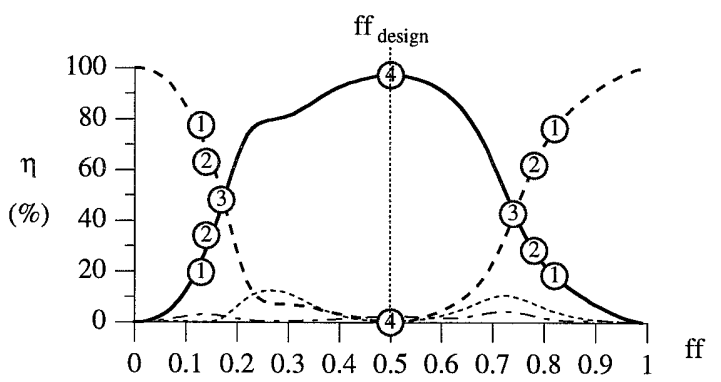

Fig. 4. Diffraction efficiencies of the $k=0,1,2$ reflected and $l=$ 1 transmitted orders for an output coupler in TE polarization as a function of (a) the relief depth, expressed as $h / \lambda$, for $\alpha$ and $f f$ constant $\left(\alpha=-30^{\circ}\right.$ and $f f=0.5$ ), (b) the grating slant angle $\alpha$, for $h$ and $f f$ constant $(h / \lambda=1.058$ and $f f=0.5)$, and (c) the fill factor $f f$, for $h$ and $\alpha$ constant $\left(h / \lambda=1.058\right.$ and $\left.\alpha=-30^{\circ}\right)$. The numbered points in (b) and (c) denote the output-grating solutions for the backplane with $N=4$ in Fig. 1(b).

\section{Synthesis of the Grating Couplers}

We begin the design procedure by concentrating on the last output coupler $(n=N)$, i.e., a grating that should deflect $100 \%$ of the incident light into the $l=$ 1 transmitted diffraction order $\left(T_{1}^{\text {out }, N}=1\right)$ and therefore out of the backplane. This element is a highefficiency beam-deflector grating whose slant angle satisfies the Bragg condition. In the search for the optimal slant angle $\alpha$, an initial estimate can therefore be given by

$$
\begin{aligned}
\alpha_{\text {init }}= & \frac{1}{2}\left[\arcsin \left(\frac{n_{0}}{\bar{n}} \sin \theta_{\text {inc }}\right)\right. \\
& \left.+\arcsin \left(\frac{n_{0}}{\bar{n}} \sin \theta_{\text {inc }}+\frac{l \lambda}{\bar{n} \Lambda_{x}}\right)\right],
\end{aligned}
$$

where we have homogenized the binary grating into a uniform slab with an effective index $\bar{n}$ using the 


\begin{tabular}{|c|c|c|c|c|}
\hline \multirow{2}{*}{$\begin{array}{c}\text { Parameters and } \\
\text { Performance Characteristics }\end{array}$} & \multicolumn{2}{|c|}{$N=4$} & \multicolumn{2}{|c|}{$N=8$} \\
\hline & $f f \leq 0.5$ & $f f \geq 0.5$ & $f f \leq 0.5$ & $f f \geq 0.5$ \\
\hline$h / \lambda$ & 1.059 & 1.057 & 1.041 & 1.046 \\
\hline$\alpha$ & $-33.50^{\circ}$ & $-24.69^{\circ}$ & $-35.55^{\circ}$ & $-24.64^{\circ}$ \\
\hline$f f$ & $f f_{1}=0.114$ & $f f_{1}=0.806$ & $f f_{1}=0.084, f f_{5}=0.109$ & $f f_{1}=0.873, f f_{5}=0.804$ \\
\hline & $f f_{2}=0.129$ & $f f_{2}=0.771$ & $f f_{2}=0.088, f f_{6}=0.122$ & $f f_{2}=0.862, f f_{6}=0.707$ \\
\hline & $f f_{3}=0.155$ & $f f_{3}=0.725$ & $f f_{3}=0.094, f f_{7}=0.144$ & $f f_{3}=0.848, f f_{7}=0.726$ \\
\hline & $f f_{4}=0.484$ & $f f_{4}=0.575$ & $f f_{4}=0.100, f f_{8}=0.504$ & $f f_{4}=0.829, f f_{8}=0.575$ \\
\hline$U(\%)$ & 1.0 & 0.4 & 2.2 & 0.5 \\
\hline$\eta(\%)$ & 94.2 & 95.6 & 92.2 & 96.4 \\
\hline$\eta_{1, \text { lost }}(\%)$ & 0.3 & 3.2 & 0.8 & 2.6 \\
\hline$\eta_{2, \text { lost }}(\%)$ & 5.0 & 0.8 & 6.1 & 0.7 \\
\hline
\end{tabular}

${ }^{a}$ Fill factors for the $N=4$ and $N=8$ gratings were $f f_{n} \leq 0.5$ and $f f_{n}=0.5$, respectively, with constant slant angles $\alpha_{n}$ for $n=1, \ldots, N$.

theory of form birefringence. In TE polarization $\bar{n}$ is determined from ${ }^{20}$

$$
\bar{n}^{2}=\frac{1}{2}\left(n_{1}{ }^{2}+n_{2}{ }^{2}\right) .
$$

This lowest-order approximation is sufficient for our purposes. From Eq. (2) and Eq. (8), where $l=1, \theta_{\text {inc }}$ $=-45^{\circ}, n_{1}=n_{0}=1.5$, and $n_{2}=1.0$, we find that $\alpha_{\text {init }}$ $=-28^{\circ}$.

The diffraction-order efficiencies were evaluated with a rigorous coupled-wave algorithm, ${ }^{21}$ thereby avoiding a decomposition of the permittivity profile into multiple slabs. We then employed parametric optimization to maximize the diffraction-order efficiency $T_{1}^{\text {out }, N}$ by tuning the relief depth, the slant angle, and the fill factor. The resulting grating parameters and diffraction-order efficiencies in the TE and TM polarizations are listed in Table 1. Although the diffraction efficiencies differ only slightly, the solution in TE polarization is preferable since the relief depth is shallower than that in TM polarization. We therefore concentrate on the design of couplers in TE polarization. In Fig. 4 , the $k=0,1,2$ and $l=1$ diffraction-order efficiencies in TE polarization are plotted around the optimum values of the three grating parameters. In each of these curves, one of the parameters, namely, $h, \alpha$, or $f f$, is varied while the two others are held constant and equal to their respective optimum values (see Table 1). The form of the curves in Fig. 4 suggests that we can attain the remaining $N-1$ output-coupler solutions by detuning the $N$ th output-coupler profile from its optimum parameters. With a constant relief depth, $h_{n}=h_{N}$, there exist two options: We may either alter $\alpha_{n}$, while holding $f f_{n}=f f_{N}$, or conversely, we can vary $f f_{n}$ by setting $\alpha_{n}=\alpha_{N}$, for $n=1, \ldots, N$. The numbered points in Figs. 4(b) and 4(c) denote the successive output gratings (for $N=4$ ) with diffraction-order efficiencies closest to those of Fig. 1(b) for different slant angles and different fill factors, respectively.

In the quest for the $N$ output couplers that can be fabricated in one etching step, we now turn our attention to the synthesis of a series of such gratings with different fill factors $f f_{n}$ but constant slant angle
$\alpha_{n}=\alpha_{N}$ for $n=1, \ldots, N$. In Fig. 4(c) there exist two separate regions containing gratings with fill factors that may match the requirements of Fig. 1(b): those with low fill factors $\left(f f_{n} \leq 0.5\right)$ and those with high fill factors $\left(f f_{n} \geq 0.5\right)$. Starting from these initial values of the fill factor $f f_{n}$, we employed a steepestdescent algorithm to minimize a quadratic merit function, defined in terms of Eqs. (3)-(6), in a manner similar to the design of grating beam splitters. ${ }^{22}$ The optimized solutions are listed in Table 2 for $N=$ 4 and $N=8$. For each of these solutions we obtain efficiencies of $\eta>90 \%$ and uniformities of $U \leq 1.0 \%$, except for $N=8$ and $\mathrm{ff}_{n} \leq 0.5$, for which we obtain $U=2.2 \%$, suggesting that this solution is not optimal
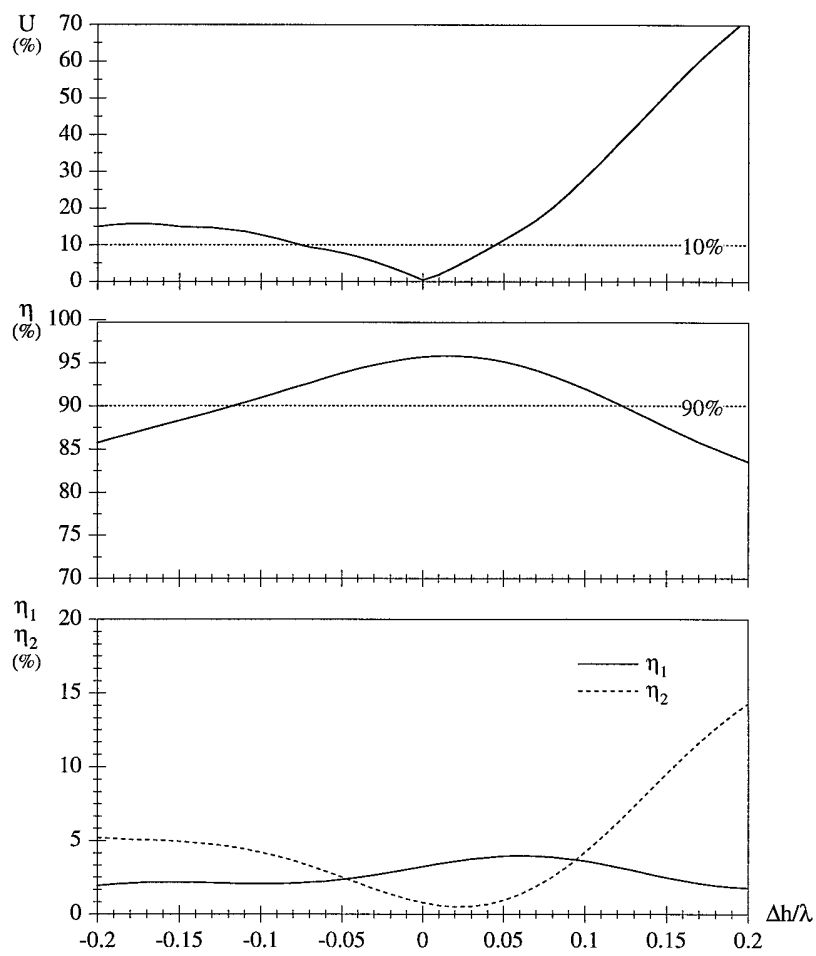

Fig. 5. Effect of a systematic error in $h / \lambda$ on the performance of the backplane: uniformity error $U$, efficiency $\eta$, and efficiency lost into the $k=1$ and $k=2$ reflected order $\eta_{1, \text { lost }}$ (solid curve) and $\eta_{2, \text { lost }}$ (dashed curve). 
since the algorithm has stagnated in a local minimum. Furthermore, we can expect extremely low values of $\eta_{2, \text { lost }}$ for a backplane employing gratings with $f f_{n} \geq 0.5$.

With our fabrication process, relief profiles with fine grooves will be more readily realizable than those with large grooves: Estimates for the realizable fill factors and slant angles are the ranges $0.2<f f_{n}<0.9$ and $0<|\alpha|<45^{\circ}$ (see Section 4). Hence, we therefore consider only solutions with $f f_{n} \geq 0.5$. During the optimization procedure we retained 11 Rayleigh orders in the calculations of the diffraction-order efficiencies. After the merit-function minimum was found, the uniformity and overall diffraction efficiency of the backplane solution converged to the fourth significant digit by the inclusion of 61 Rayleigh orders.

Now that we have synthesized a set of $N$ outputcoupler gratings that can, in principle, be realized in one etching step, it remains to specify an input coupler. In exploring the possibility of also using a slanted grating, we notice that, for the specific backplane configuration with $M=0$ [Fig. 1(b)], we require an input coupler that operates as a high-efficiency beam-deflector element at normal incidence. By the reciprocity theorem ${ }^{3}$ we have already specified the profile of this coupler, which is the mirror image (with respect to $z$ ) of the last output-coupler profile listed in Table 1. However, the addition of this element to the backplane implies an extra etching step over and above the first etch required for the output couplers. Nonetheless, for a backplane with one input coupler and $M+N$ output couplers, we have reduced the

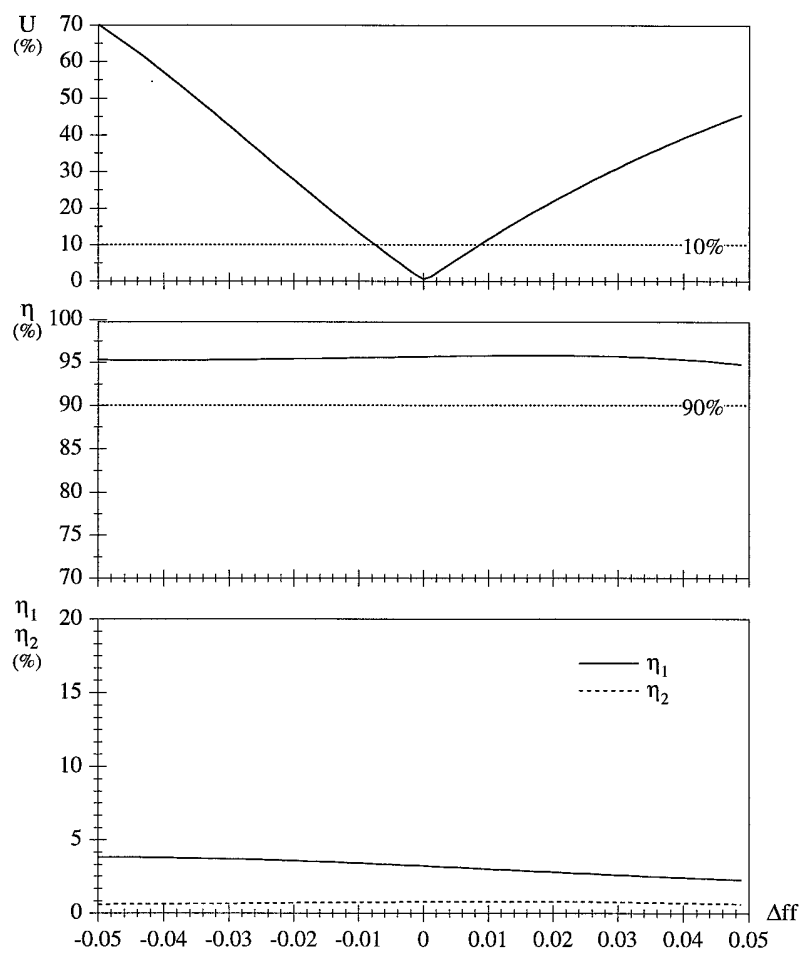

Fig. 6. Effect of a systematic error in the fill factor $f f$ on the performance of the backplane. total number of etching steps from 1(input coupler) + $M+N$, as would be required for output couplers with different $\alpha_{n},{ }^{16,17}$ to 1 (input coupler) $+1(\mathrm{IF} M \neq 0)+$ $1($ IF $N \neq 0) \leq 3$, with output couplers with different $f f_{n}$.

\section{Fabrication-Error Analysis}

Direct electron-beam writing offers a realistic means of patterning the fine grating structures that were presented in Section 3. Despite the high resolution of this particular plotting technique, it is inevitable that the entire fabrication process (initial patterning, pattern transfer, etching, and possible replication) will introduce some form of inaccuracy to the grating profiles. In this section, we examine the sensitivity of the solution of $N=4$ output couplers with constant slant angle and different high fill factors $f f \geq 0.5$ (the grating parameters are given in the second column of results in Table 2) to some of the more common manufacturing errors, i.e., deviation from the ideal relief depth, fill factors, and slant angle and incorrectly shaped groove walls. The analysis of the two latter types of error is particularly important since it can be expected to be difficult to control the slant angle when employing RIE to generate slanted structures. ${ }^{23-25}$

To draw a comparison with the results that follow, it should be noted that a backplane composed of $N=$ 4 identical output couplers that consist of singlegrooved binary lamellar reflection gratings, ${ }^{14}$ and one which is manufactured perfectly, will have values of $U=33.8 \%, \eta=52.4 \%$, and $\eta_{2, \text { lost }}=7.3 \%$. Our intention, therefore, is to determine the expected performance gain over these parameters for a backplane

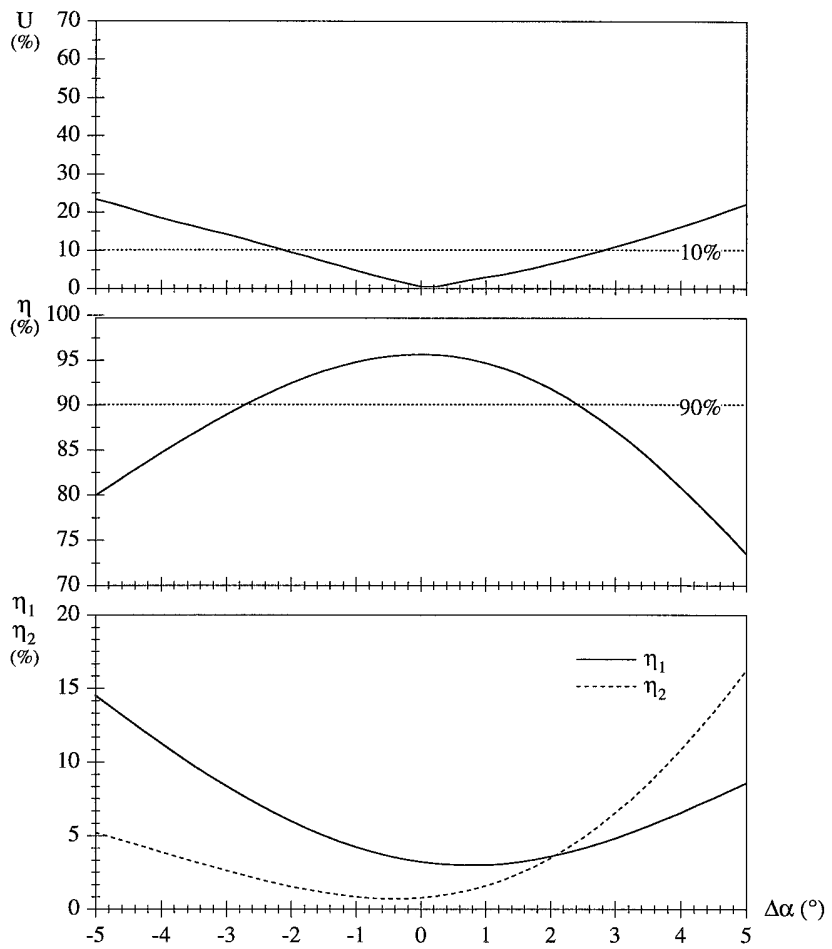

Fig. 7. Effect of a systematic error in the slant angle $\alpha$ on the performance of the backplane. 
(a)

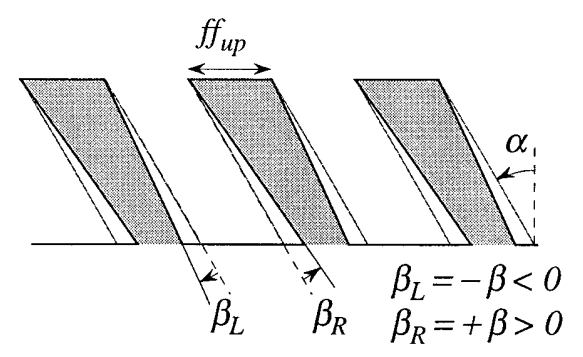

(b)

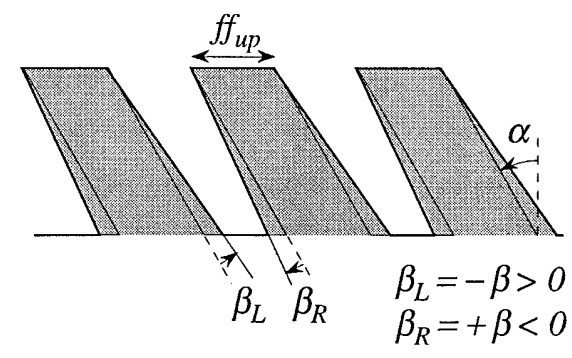

(c)

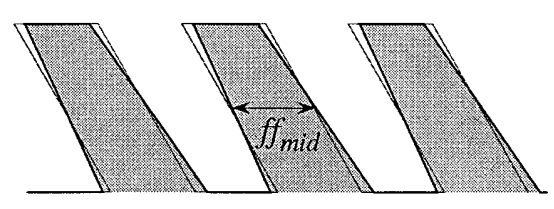

(d)

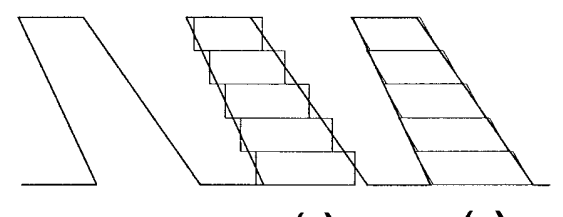

(1)

(2)

Fig. 8. Slanted binary gratings with nonparallel groove walls: (a) undercutting $(\beta>0)$, (b) overcutting $(\beta<0)$, (c) overcut profile with compensation, and (d) approximation with stacked binary lamellar profiles and varying fill factors with (1) nonslanted and (2) slanted sections.

composed of $N=4$ different output couplers and in the presence of manufacturing errors.

In what follows the backplane figures of merit, given by Eqs. (3)-(6), are presented as a function of the magnitude of a particular fabrication error. In Fig. 5 is shown the effect on performance of the backplane solution of a systematic error in the relief depth $h$, expressed as $\Delta h / \lambda$. The uniformity error is less sensitive to negative values of $\Delta h / \lambda$ than it is to positive values of $\Delta h / \lambda$. The overall efficiency $\eta$ drops by some $10 \%$ with $\Delta h / \lambda= \pm 0.2$ and $\eta_{1 \text {,lost }}$ does not vary noticeably in the interval, whereas $\eta_{2, \text { lost }}$ dips at values of $h / \lambda$ close to the optimum. For a value of $\Delta h / \lambda=0.05$ we find that $U=11.3 \%, \eta=$ $91.1 \%, \eta_{1, \text { lost }}=4.0 \%$, and $\eta_{2, \text { lost }}=1.0 \%$.

The consequences of errors in the fill factors and nonideal slant angles are shown in Figs. 6 and 7 . The uniformity error is extremely sensitive to inac- curacies in the fill factors, whereas the overall diffraction efficiency is unaltered. This surprising effect arises from the symmetry of the $l=1$ and $k=$ 0 diffraction efficiencies [see Fig. 4(c)], which leads to an exact compensation of the four output couplers' contribution to the total efficiency $\eta$, even if they are not equal. An error of $U=10 \%$ occurs for $\Delta f f=$ \pm 0.009 , at which point $\eta=95.7 \%, \eta_{1, \text { lost }}=3.0 \%$, and $\eta_{2, \text { lost }}=1.0 \%$. Errors in the slant angle as large as $\Delta \alpha= \pm 5^{\circ}$ relate to a uniformity error of $U=24 \%$. For these errors we find that $U=10 \%$ when $\Delta \alpha=$ $-2.25^{\circ}$ and $\eta=91.6 \%, \eta_{1, \text { lost }}=5.2 \%$, and $\eta_{2, \text { lost }}=$ $1.8 \%$.

If RIE or focused ion-beam milling are employed to generate slanted surface-relief profiles, ${ }^{17}$ we can expect to obtain features with sidewalls that are not parallel but are either undercut [Fig. 8(a)] or overcut $^{23}$ [Fig. 8(b)]. We denote the change in the angle of the groove walls by $\beta$. A positive value of $\beta$ implies undercutting [Fig. 8(a)], whereas a negative value corresponds to overcutting [Fig. 8(b)].

We attempted to compensate for the effects of over- and undercutting by ensuring that the fill factor in the middle of the groove $f f_{\text {mid }}$ is equal to the design value 22 [Fig. 8(c)]. The groove walls then pivot about this center position as $\beta$ varies. A groove profile without compensation implies that the fill factor at the top of the grooves $f f_{\text {up }}$ has the ideal value [Figs. 8(a) and 8(b)]. We analyzed the effects of over- and undercutting using electromagnetic grating theory, ${ }^{21}$ in which we approximated gratings with nonparallel groove walls by stacking multiple slanted binary profiles with varying fill

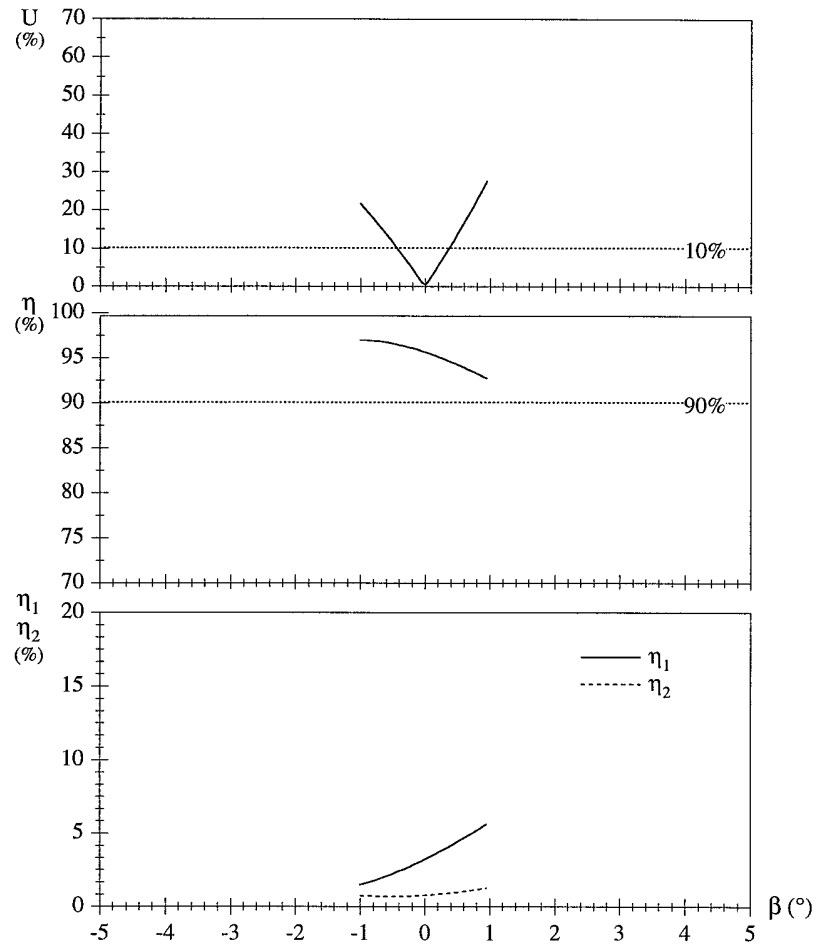

Fig. 9. Effects of overcutting $(\beta<0)$ and undercutting $(\beta>0)$, without compensation, on the performance of the backplane. 

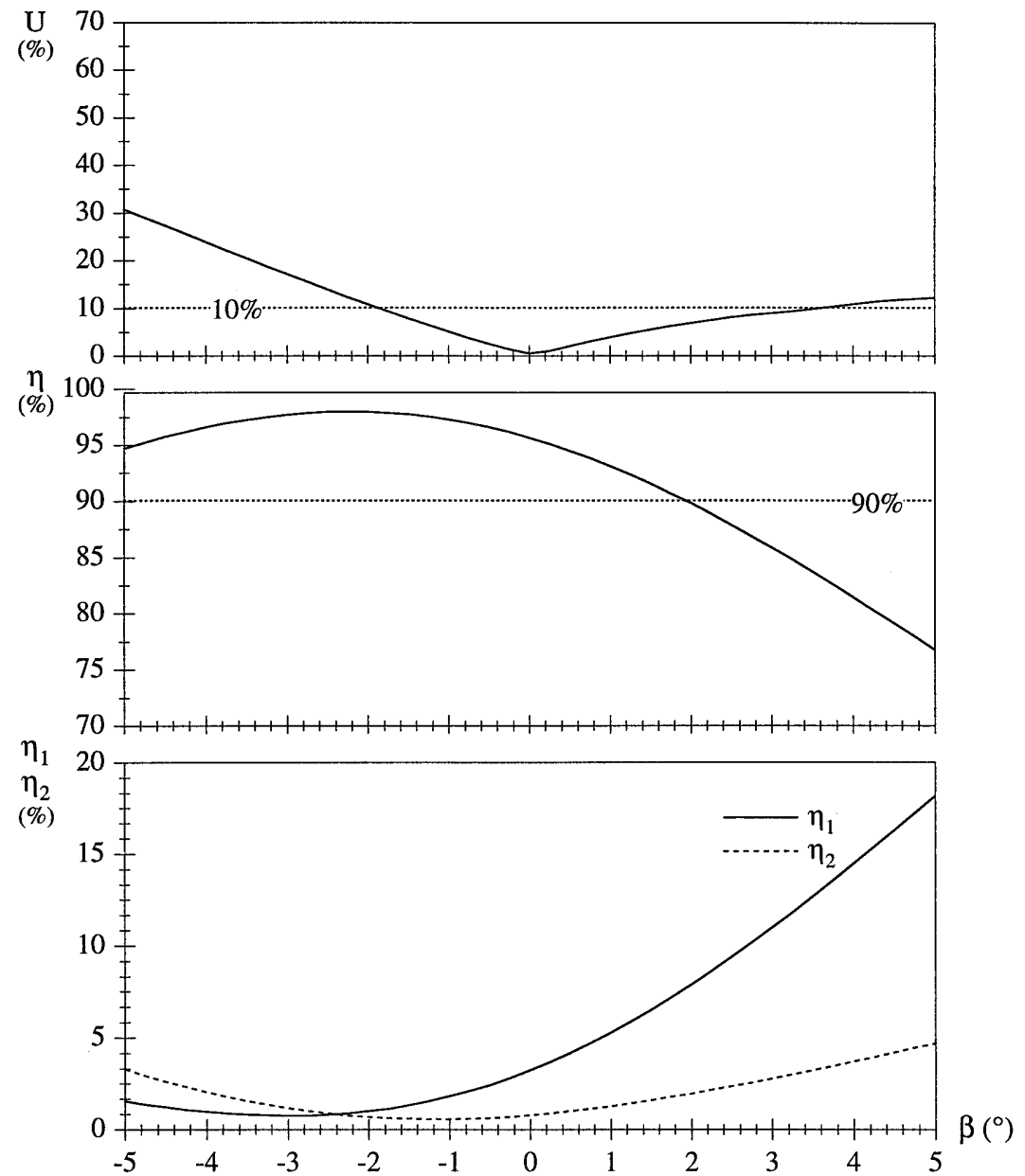

Fig. 10. Effect of undercutting $(\beta>0)$ and overcutting $(\beta<0)$, with compensation, on the performance of the backplane.

factors [Fig. 8(d), part (2)]. Convergence of the diffraction-order efficiencies can then be achieved with half as many slices compared with the approximation shown in Fig. 8(d), part (1).

The effects of this type of error for noncompensated and compensated profiles are shown in Figs. 9 and 10, respectively, with the limits for $\beta$ being determined by intersecting groove walls. The introduction of compensation into the profiles indeed reduces the sensitivity of the backplane solution to this type of error: In effect we ensure that the amount of material in the grating profile remains equal to that of the parallel-grooved grating. Our initial experimental results (see Section 4) suggest that we can expect an error of $\beta \approx 1.6^{\circ}$, i.e., undercutting. Compensation of the groove profiles is therefore essential.

In this analysis we have assumed that only one specific fabrication error is present in the grating profiles. If an equal error is introduced into each of the $n=1, \ldots, N$ couplers, we require an accuracy of at least $\Delta h / \lambda=0.05, \Delta f f= \pm 0.009$, and $|\Delta \alpha|=2.25^{\circ}$ and compensation of the groove walls to obtain an $N=4$ backplane with values $U \leq 10 \%, \eta \geq 90 \%$, and $\eta_{1, \text { lost }}$ and $\eta_{2, \text { lost }}$ as low as possible.

\section{Experimental Verification}

For an initial verification of our design process, we elected to realize the output-coupler solution in TE polarization (Table 1) for an operating wavelength of $\lambda=0.633 \mu \mathrm{m}$. The slanted surface-relief grating was realized in photoresist by use of direct electronbeam writing and RIE with a procedure similar to that used for manufacturing high-aspect-ratio effective-index diffractive elements ${ }^{26}$; it is as follows: Photoresist (with a refractive index of $n_{1}=1.6$, which was measured interferometrically) was spin coated on a $220-\mu \mathrm{m}$-thick glass wafer (with a refractive index of 1.52) to a depth of $1.0 \mu \mathrm{m}$. A $0.1-\mu \mathrm{m}$-thick layer of metal was then deposited onto the surface of the photoresist, and the ensemble was coated with $0.15-\mu \mathrm{m}$ poly(methyl methacrelate) (PMMA). The high-frequency structure was then patterned over a 2 $\mathrm{mm} \times 2 \mathrm{~mm}$ area by use of a Jeol Model JBX5D2U electron-beam writer with an accelerating voltage of $50 \mathrm{kV}$ and a beam current of $10 \mathrm{nA}$, producing a spot diameter of $100 \mathrm{~nm}$. After development of the PMMA, the metal layer was chemically etched. The PMMA was then removed, leaving the metal layer as 


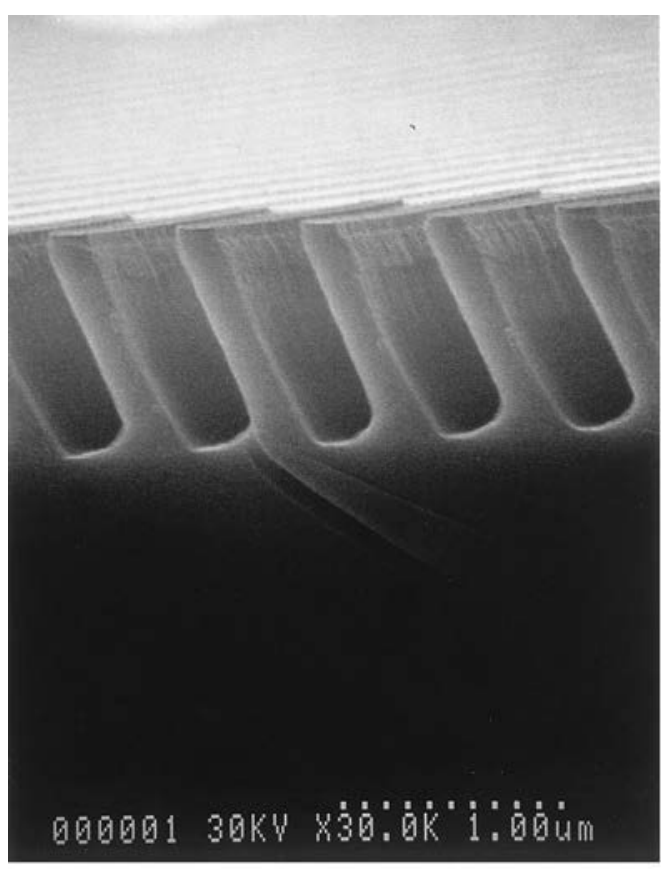

(a)

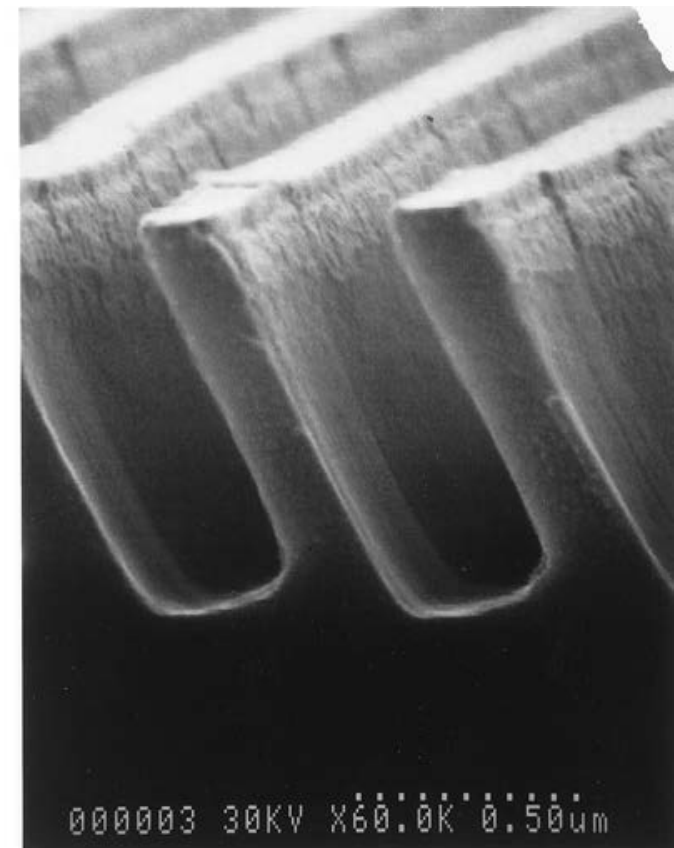

(b)

Fig. 11. SEM micrographs of a slanted surface-relief grating in photoresist generated by use of electron-beam writing and RIE: (a) cross section at the nonoblique perspective of the slanted-grating profile and (b) a close-up showing the rounded edges and slight undercut.

the mask. A standard RIE chamber was employed to generate the slanted surface-relief profiles. Here, the substrate was oriented by approximately $-30^{\circ}$ to the electrode plates, while ensuring that the grooves were parallel to the edge of the holder.

A scanning electron microscope (SEM) micrograph of the resulting grating is shown in Fig. 11. We measured from Fig. 11(a) that the slant angle had a slant angle of $\alpha \approx-22^{\circ}$. The fill factor and the relief depth were determined in the following manner: An accurate scale for the SEM micrograph was established by use of the grating period as a standard length. This was deemed possible since the resolution of the electron-beam writer is $2.5 \mathrm{~nm}$, so the grating period is $\Lambda_{x}=0.598 \pm 0.0025 \mu \mathrm{m}$. From this scale these parameters were straightforwardly calculated to be ff $\approx 0.35$ and $h \approx 1.0 \mu \mathrm{m}$. The difference between the grating slant angle and that of the substrate holder is $\approx 8^{\circ}$. We expect that this is due to electric-field inhomogeneities as the substrate holder extends into the plasma dark space.

We are currently examining different techniques to obtain more accurate control of the slant angle ${ }^{23-25}$ and the fill factor to achieve the required precision as determined in Section 3. The grating realized in photoresist does not correspond to optimal values of the parameters determined in Subsection 2.C (Table 1 and Fig. 4), therefore we cannot expect the highest efficiency for the resulting output coupler.

The $k=0,1,2$ reflected and $l=1$ transmitted diffraction orders were recalculated with the experimental values of the parameters (i.e., grating period, fill factor, slant angle, and refractive indices of the photoresist and substrate) and are plotted in
Fig. 12 as a function of the relief depth $h$. A high efficiency can be expected for relief depths in the range $0.3 \mu \mathrm{m}<h<0.6 \mu \mathrm{m}$. In an attempt to obtain a relief depth within this region, another grating was prepared (once again with a resist with a thickness of $1 \mu \mathrm{m}$ ), and the etch time was calibrated with the aid of the SEM micrograph shown in Fig. 11.

We characterized this grating while it operated as both an input and an output coupler at the design wavelength of $\lambda=0.633 \mu \mathrm{m}$. For measuring the reflected and transmitted diffraction-order efficiencies, the grating substrate was index matched onto a parallelogramic prism with a $45^{\circ}$ angle. Optically cementing antireflection coatings onto three faces of the prism (corresponding to the input beam and the

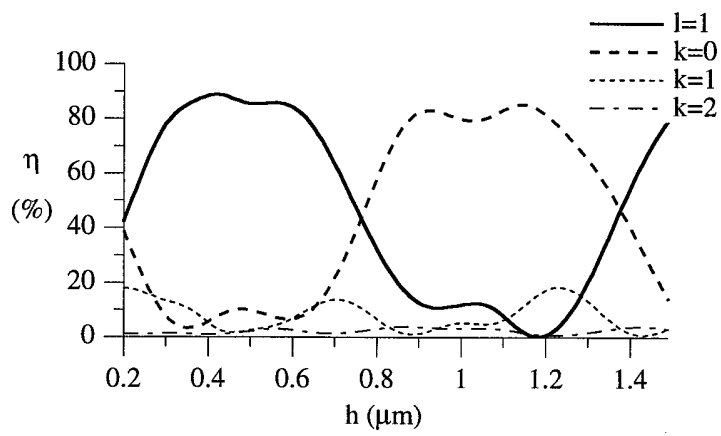

Fig. 12. Calculated diffraction efficiencies for an output coupler as a function of $h$ (in micrometers). Other grating parameters are $\Lambda_{x}=0.598 \mu \mathrm{m}, \alpha=-22^{\circ}, f f \approx 0.35, n_{0}=1.52, n_{1}=1.60, n_{2}=n_{3}$ $=1, \theta_{\text {inc }}=-44.03^{\circ}$. 
Table 3. Experimental and Theoretical Diffraction Efficiencies for the Fabricated Slanted Grating Acting as Output Coupler or Input Coupler

\begin{tabular}{|c|c|c|c|}
\hline \multirow[b]{2}{*}{ Coupler Mode } & \multirow{2}{*}{$\begin{array}{l}\text { Diffracted } \\
\text { Order }(\%)\end{array}$} & \multicolumn{2}{|c|}{ Results } \\
\hline & & Experimental & Theoretical \\
\hline \multirow[t]{4}{*}{ Output } & $T_{1}^{\text {out }}$ & 46 & 48.6 \\
\hline & $R_{0}^{\text {out }}$ & 30 & 42.0 \\
\hline & $R_{1}^{\text {out }}$ & 4 & 2.0 \\
\hline & $R_{2}^{\text {out }}$ & 7 & 7.4 \\
\hline \multirow[t]{4}{*}{ Input } & $T_{-1}^{\mathrm{in}}$ & 46 & 49.0 \\
\hline & $T_{0}^{\mathrm{in}}$ & 33 & 38.1 \\
\hline & $T_{1}^{\text {in }}$ & 8 & 11.4 \\
\hline & $R_{0}^{\text {in }}$ & 3 & 1.5 \\
\hline
\end{tabular}

reflected diffraction orders in the output mode) avoided multiple reflections in the prism.

The experimentally measured diffraction-order efficiencies are given in Table 3 . We have included the best-fitting numerical values for an ideal parallelwalled slanted grating, corresponding to an estimated relief depth of $h=0.75 \mu \mathrm{m}$. In this analysis we included the $0.25-\mu \mathrm{m}$ unetched resist layer underneath the grating structure. Some $13 \%$ and $10 \%$ of the incident light is unaccounted for when the grating operates as an output or an input coupler, respectively. This can be explained partially by slight absorption in the photoresist and also by the existence of extra orders around the measured one that are caused by stitching errors (we step and repeat at $80-\mu \mathrm{m}$ intervals in both directions).

The experimental and theoretical results listed in Table 3 are in reasonable agreement, although a more accurate analysis would require a SEM micrograph, for which we would need to gold-coat and cleave the grating, rendering it useless. Nonetheless, we have clearly demonstrated with these initial results the prospects of such elements and, furthermore, that such binary slanted surface-relief profiles are indeed compatible with state-of-the-art microlithographic fabrication techniques.

\section{Conclusion and Discussion}

In conclusion, we have investigated the concept of binary slanted surface-relief gratings for input and output coupling in a planar optical backplane. Parametric optimization was used to design a backplane composed of these gratings with a high efficiency and low uniformity error and loss into the $k=$ 1 and $k=2$ reflected orders. We demonstrated two configurations of output couplers: Either the slant angle of the individual gratings varies while the fill factor and relief depth remain constant, or the slant angle and relief depth are constant while the fill factor varies. It is this latter possibility that enables a set of $N$ output-coupler gratings to be manufactured in truly one lithographic etching step.

In light of the fabrication-error analysis and, moreover, of our initial experimental results, it seems reasonable to assume that the realization of a planar optical interconnect consisting of multiple binary slanted gratings is indeed feasible. Furthermore, replication techniques such as UV embossing could be employed to copy these slanted gratings, ${ }^{27}$ leading to the eventual mass production of such a planar optical interconnect. In future systems the flexibility of such interconnects may also be enhanced by multiple connections at each output coupler. The technique of pulse-frequency modulation ${ }^{28}$ of a highfrequency carrier grating can be employed to encode optical functions such as fan-out and focusing, ${ }^{29}$ thereby increasing the interconnection density and reducing the complexity of the receiver optics.

J. M. Miller thanks his family, friends, and colleagues for their continued support, which has enabled the completion of this study. He is grateful to P. Ehbets for fruitful discussions and D. Joyeux for measuring the refractive index of the photoresist. A Human Capital and Mobility fellowship from the EC is acknowledged. This project was partially supported by Ultimatech, Centre National de la Recherche Scientifique, France. We thank P. Pääkkönen for his help in the preparation of the manuscript. We also thank the reviewers for their suggestions on improving this manuscript.

\section{References}

1. J. Jahns and A. Huang, "Planar integration of free-space optical components," Appl. Opt. 28, 1602-1605 (1989).

2. S. J. Walker and J. Jahns, "Optical clock distribution using integrated free-space optics," Opt. Commun. 90, 359-371 (1992).

3. R. Petit, ed., Electromagnetic Theory of Gratings, Vol. 22 of Topics in Current Physics (Springer-Verlag, Berlin, 1980).

4. E. Noponen, A. Vasara, J. Turunen, J. M. Miller, and M. R. Taghizadeh, "Synthetic diffractive optics in the resonance domain," J. Opt. Soc. Am. A 9, 1206-1213 (1992).

5. A. A. Friesem and Y. Amitai, "Planar diffractive elements for compact optics," in Trends in Optics: Research, Developments and Applications, A. Consortini, ed. (Academic, San Diego, Calif., 1996), pp. 125-144.

6. J. Turunen and F. Wyrowski, "Diffractive optics: from promise to fruition," in Trends in Optics: Research, Developments and Applications, A. Consortini, ed. (Academic, San Diego, Calif., 1996), pp. 111-123.

7. J. M. Miller, J. Turunen, E. Noponen, A. Vasara, and M. R. Taghizadeh, "Rigorous modal theory for multiply grooved lamellar gratings," Opt. Commun. 111, 526-535 (1994).

8. J. Saarinen, E. Noponen, J. Turunen, T. Suhara, and H. Nishihara, "Asymmetric beam deflection by doubly grooved binary gratings," Appl. Opt. 33, 2401-2405 (1995).

9. E. Noponen, J. Turunen, and A. Vasara, "Parametric optimization of multilevel diffractive optical elements by electromagnetic theory," Appl. Opt. 31, 5910-5912 (1992).

10. R. K. Kostuk, Y.-T. Huang, D. Hetherington, and M. Kato, "Reducing alignment and chromatic sensitivity of holographic interconnects with substrate-mode holograms," Appl. Opt. 28, 4939-4944 (1989).

11. J. H. Yeh and R. K. Kostuk, "Free-space holographic optical interconnects for board-to-board and chip-to-chip interconnections," Opt. Lett. 21, 1274-1276 (1996).

12. S. Natarajan, C. Zhao, and R. T. Chen, "Bi-directional optical backplane bus for general purpose multi-processor board-toboard optoelectronic interconnects," J. Lightwave Technol. 13, 1031-1040 (1995).

13. T. Nakaya, Y. Katoh, T. Kubota, and M. Takeda, "Diffraction 
efficiency of a grating coupler for an array illuminator," Appl. Opt. 35, 3891-3898 (1996).

14. J. W. Parker, "Optical interconnection for advanced processor systems: a review of the ESPRIT II OLIVES program," J. Lightwave Technol. 9, 1764-1773 (1991).

15. S. Paineau, J.-P. Ghesquiers, M. Charrier, and T. Lemoine, "Clock distribution by holographic optical backplane in massively parallel processor architecture," in Free Space MicroOptical Systems (European Optical Society, Engelberg, Switzerland, 1996), pp. 52-53.

16. J. M. Miller, N. de Beaucoudrey, P. Chavel, M. François, and J. Turunen, "Inclined binary high-frequency gratings for an optical backplane interconnect," in Proceedings of a Workshop on Diffractive Optics, Prague, Czech Republic, August 1995, pp. 33-34.

17. N. de Beaucoudrey, J. M. Miller, P. Chavel, and J. Turunen, "On the design and fabrication of high efficiency inclined binary high frequency gratings," in Specification, Production, and Testing of Optical Components and Systems, A. E. Gee and J. Houee, eds., Proc. SPIE 2775, 533-536 (1996).

18. M. Takeda and T. Kubota, "Integrated optic array illuminator: a design for efficient and uniform power distribution," Appl. Opt. 30, 1090-1095 (1991).

19. D. Maystre, ed., Selected Papers on Diffraction Gratings, Vol. M583 of SPIE Milestone Series (SPIE Press, Bellingham, Wash., 1993), pp. xvii-xxx.

20. M. Born and E. Wolf, Principles of Optics, 6th ed. (Pergamon, London, 1989), pp. 705-708.
21. N. Chateau and J.-P. Hugonin, "Algorithm for the rigorous coupled-wave analysis of grating diffraction," J. Opt. Soc. Am. A 11, 1321-1331 (1994).

22. A. Vasara, M. R. Taghizadeh, J. Turunen, J. Westerholm, E. Noponen, H. Ichikawa, J. M. Miller, T. Jaakkola, and S. Kuisma, "Binary surface-relief gratings for array illumination in digital optics," Appl. Opt. 31, 3320-3336 (1992).

23. L. A. Coldren and J. A. Rentschler, "Directional reactive-ion etching of InP with $\mathrm{Cl}_{2}$ containing gases," J. Vac. Sci. Technol. 19, 225-230 (1981).

24. T. Takamori, L. A. Coldren, and J. L. Merz, "Angled etching of GaAs/AlGaAs by conventional $\mathrm{Cl}_{2}$ reactive ion etching," Appl. Phys. Lett. 53, 2549-2551 (1988).

25. B. Jacobs and R. Zengerle, "Reactive ion etching of sloped sidewalls for surface emitting structures using a shadow mask technique," J. Vac. Sci. Technol. B 14, 2537-2542 (1996).

26. J. M. Miller, N. de Beaucoudrey, P. Chavel, E. Cambril, and H. Launois, "Synthesis of a subwavelength-pulse-width spatially modulated array illuminator for $0.633 \mu \mathrm{m}$," Opt. Lett. 21, 1399-1401 (1996).

27. J. Bell, "Market misses message about diffractive optics," Opto Laser Europe 21-28 (March 1997).

28. E. Tervonen, J. Turunen, and J. Pekola, "Pulse-frequencymodulated high-frequency-carrier diffractive elements for pattern projection," Opt. Eng. 33, 2579-2587 (1994).

29. M. Li, A. Larsson, N. Eriksson, and M. Hagberg, "Continuouslevel phase-only computer-generated hologram realized by dislocated binary gratings," Opt. Lett. 21, 1516-1518 (1996). 